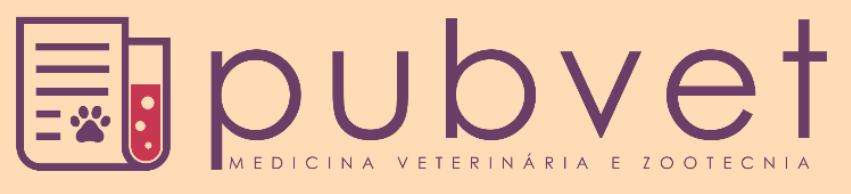

https://doi.org/10.31533/pubvet.v16n01a1017.1-8

\title{
Distocia em Jabuti-piranga (Chelonoidis carbonaria) em cativeiro: Relato de caso
}

\author{
Bianca Sandrin Saim $^{1 *}$, José Carlos Roble Júnior ${ }^{2 \theta}$, Paulo Rogério Mangini ${ }^{2} \theta$, Antonella Souza Mattei ${ }^{3 \theta}$ \\ ${ }^{I}$ Discente do Curso de Medicina Veterinária da Universidade de Caxias do Sul. Caxias do Sul/RS, Brasil \\ ${ }^{2}$ Médico Veterinário na Clínica Vida Livre Medicina de Animais Selvagens. Curitiba/PR, Brasil \\ ${ }^{3}$ Docente do Curso de Medicina Veterinária da Universidade de Caxias do Sul. Caxias do Sul/RS, Brasil \\ *Autor para correspondência, E-mail: biancasaim@hotmail.com
}

\begin{abstract}
Resumo. Os jabutis são quelônios terrestres pertencentes à ordem dos Testudines. Cada vez mais existem répteis criados como animais de estimação, e muitas vezes por causa da falta de orientação e conhecimento dos tutores sobre a biologia e fisiologia destes animais, várias doenças ocorrem por manejo inadequado nesta espécie. Um exemplo disso é a retenção de ovos ou distocia, a qual se trata de uma afecção comumente relatada em répteis criados em cativeiro. Nos casos em que houver suspeita de distocia, é importante realizar a radiografia como exame complementar para auxiliar no diagnóstico de retenção de ovos. Para o tratamento, quando o manejo clínico não invasivo falhar na solução do problema, intervenções cirúrgicas deverão ser consideradas. O objetivo deste trabalho foi relatar um caso de distocia em jabuti-piranga em cativeiro que inicialmente foi instituído o tratamento medicamentoso através da administração de ocitocina e borogluconato de cálcio. Devido à falta de resposta, a paciente foi submetida ao procedimento cirúrgico, obtendo assim, um resultado positivo.
\end{abstract}

Palavras chave: Celiotomia, distocia, ocitocina, ovariosalpingooviductotomia

\section{Dystocia in Red-footed tortoise (Chelonoidis carbonaria) in captivity: Case report}

\begin{abstract}
Tortoises are terrestrial chelonians that belong to the order of Testudines. More and more reptiles are being raised as pets and the lack of guidance and knowledge of the owners about the biology and physiology of those animals resulting in an inappropriate management of the species are often the cause of several diseases that affect these animals. An example of this is the egg retention or dystocia, which is a condition commonly reported in reptiles bred in captivity. In cases where dystocia is suspected it is important to perform radiography as a complementary test to aid in the diagnosis of retained eggs. If noninvasive clinical management fails to treat the condition, surgical interventions should be considered. The purpose of this study was to report a case of dystocia in captive tortoise, which initially received drug treatment through the administration of oxytocin and calcium borogluconate. Due to the lack of a response to the treatment, the animal was submitted to a surgical procedure obtaining a positive result.
\end{abstract}

Keywords: Celiotomy, dystocia, oxytocin, ovariosalpingooviductotomy

\section{Introdução}

Hoje em dia observa-se um aumento no interesse em ter répteis como animais de companhia e, consequentemente, uma maior importância nos estudos dessa classe. Os jabutis são quelônios terrestres pertencentes à família Testudinidae, ordem dos Testudines (Pritchard et al., 1990). Possuem corpo 
robusto, carapaça arqueada, membros locomotores fortes apropriados para suportar o peso do casco e caminhar em ambientes rústicos (Cubas et al., 2014).

A reprodução do jabuti é ovípara, tendo como fatores importantes na determinação de sua sazonalidade o fotoperíodo, a temperatura, o ciclo térmico e a oferta de alimentos. A maturidade sexual ocorre por volta dos cinco a sete anos, atingindo o número de seis a 20 ovos por postura, a qual ocorre de cinco a seis semanas após o acasalamento. Os ovos permanecem incubados de seis a nove meses numa temperatura entre 28 a $31^{\circ} \mathrm{C}$ (Cubas et al., 2014). A reprodução da espécie acontece no período de agosto a janeiro, com maior frequência entre setembro a novembro, sendo de fácil adaptação reprodutiva em cativeiro (Jepson, 2010; Vogt et al., 2009).

A distocia trata-se de uma afecção comumente relatada em répteis criados em cativeiro podendo estar associada a erros de manejo como temperatura inadequada, ambiente errado e alimentação deficiente (Denardo, 1996). Nos casos em que houver suspeita de distocia, deve-se recorrer aos exames complementares como a radiografia para auxiliar no diagnóstico de retenção de ovos (Jepson, 2010; Vogt et al., 2009). Para o tratamento, quando as técnicas clínicas não invasivas falharem na solução do problema, intervenções cirúrgicas deverão ser consideradas (Divers \& Mader, 2005).

O objetivo deste trabalho foi relatar um caso de distocia em jabuti-piranga atendida em uma clínica veterinária particular na cidade de Curitiba/PR, sendo instituído primeiramente o manejo clínico e, após a falta de resposta terapêutica, foi realizada a manobra cirúrgica, com resolução do caso.

\section{Relato de caso}

Chegou para atendimento na Clínica Vida Livre Medicina de Animais Selvagens em Curitiba/PR, em março de 2021, uma jabuti-piranga (Chelonoidis carbonaria), fêmea, de 22 anos, com massa corporal de $8,3 \mathrm{~kg}$. Durante a anamnese a tutora relatou que no dia anterior à consulta a paciente realizou a postura de um ovo pela primeira vez na vida e sem presença de macho no ambiente. Após a postura, a fêmea apresentou sangramento cloacal e contrações que continuaram por mais de uma hora. Além disso, entrou em sua toca e ficou sem se alimentar até o dia seguinte. A tutora relatou, também, que o animal ficava solto no jardim e possuía uma toca com lâmpada incandescente. A alimentação fornecida era ração comercial Reptolife ${ }^{\circledR}$ de jabuti, sendo adicionado camarão e frutas à refeição. Alegou que o animal nunca havia adoecido anteriormente.

Ao exame físico, a paciente permaneceu ativa, sem alterações na palpação da cavidade celmática e apresentava sangramento na região cloacal. O ovo eliminado foi trazido pela tutora, o qual demonstrava formato relativamente grande comparado ao tamanho do animal e casca regular, porém bastante espessa e com presença de sangue (Figura 1).

Com suspeita de distocia, realizou-se exame radiográfico como método auxiliar de diagnóstico. As projeções utilizadas foram dorsoventral (Figura 2A) e latero-lateral (Figura 2B), onde pode-se observar imagem compatível com presença de ovo, contabilizando seis estruturas ovoides.

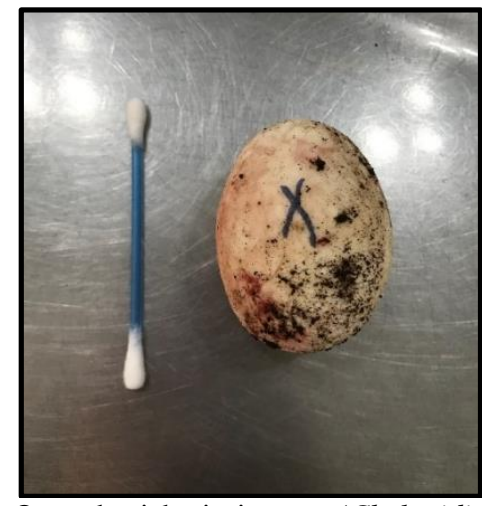

Figura 1. Ovo de jabuti-piranga (Chelonidis carbonaria) medindo em torno de $6 \mathrm{~cm}$, sendo comparado ao tamanho de uma haste flexível de algodão, apresentando casca espessa, formato regular e com presença de sangue no exterior.

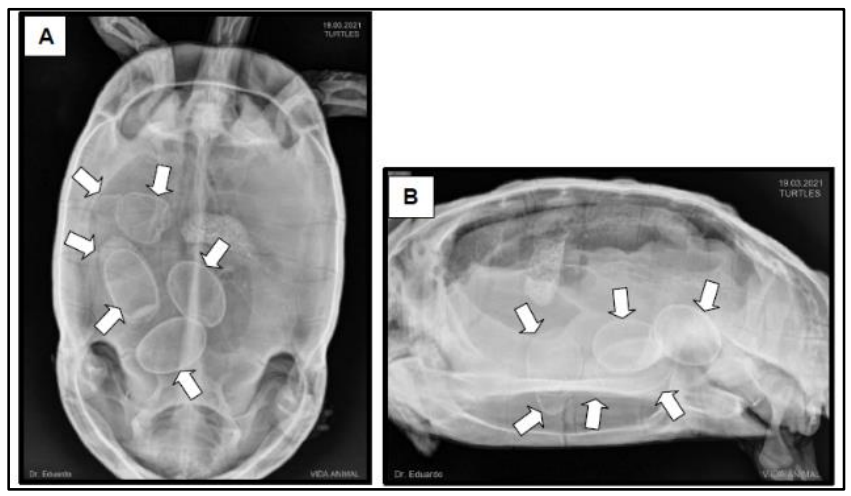

Figura 2. Exame radiográfico de jabuti-piranga (Chelonoidis carbonaria) com suspeita de distocia atendida em uma clínica veterinária particular apresentando 6 estruturas ovoides (setas). A) Posição dorsoventral B) Posição laterolateral. Fonte: Eduardo Koerbel (2021). 
A paciente foi internada para iniciar tratamento clínico, sendo considerada também uma possível intervenção cirúrgica, caracterizando um prognóstico reservado.

Durante o tratamento hospitalar foi administrada ocitocina (1 UI/ $/ \mathrm{kg}$ ), por via intramuscular, a cada três dias, totalizando três aplicações e fluidoterapia com solução de Ringer Lactato no volume de $10 \%$ do peso corporal do animal, ou seja, $40 \mathrm{~mL}$, por via subcutânea, a cada 2 dias, totalizando 9 aplicações. Nessa fluidoterapia foi adicionado borogluconato de cálcio $(10 \mathrm{mg} / \mathrm{kg})$, enrofloxacina $10 \%(5 \mathrm{mg} / \mathrm{kg})$ e uma solução contendo aminoácidos, vitaminas do complexo B e glicose (Mercepton®) (dose empírica de $5 \mathrm{~mL} / \mathrm{animal}$ ).

Ao longo do tratamento, a paciente permaneceu em local aquecido no internamento de répteis durante a noite, enquanto que, no período do dia ficava solta em ambiente externo, onde havia terra para garantir um local adequado caso houvesse postura.

Após uma semana de internação e sem sinal de ovoposição, foi realizado um novo exame radiográfico, nas mesmas projeções já descritas anteriormente. Ao repetir a radiografia, foi visto que praticamente não houve mudanças no posicionamento dos ovos, no entanto, verificou-se a presença de gases em alças intestinais (Figura 3). Diante disso, iniciou-se a administração de metronidazol (12 $\mathrm{mg} / \mathrm{kg}$ ) por via subcutânea, uma vez ao dia durante 5 dias.

Durante o período de internação o animal permaneceu ativo e comendo normalmente, porém, diante da falta de resposta ao tratamento medicamentoso após 20 dias de terapia, optou-se pela realização de procedimento cirúrgico para retirada dos ovos, sendo realizada a celiotomia, seguida de ovariosalpingooviductotomia.

Para a cirurgia, o animal foi sedado utilizando como medicação pré-anestésica (MPA) cetamina (20 $\mathrm{mg} / \mathrm{kg})$, midazolam $(2 \mathrm{mg} / \mathrm{kg})$, butorfanol $(1,5 \mathrm{mg} / \mathrm{kg})$ e dexmedetomidina $(0,1 \mathrm{mg} / \mathrm{kg})$, sendo todos aplicados por via intramuscular. A indução foi realizada através da administração de propofol $(5 \mathrm{mg} / \mathrm{kg})$ por via intravenosa, após uma hora da aplicação da MPA. A paciente foi entubada para manutenção dos movimentos respiratórios durante o procedimento; porém, no decorrer da cirurgia demonstrou estímulos respiratórios próprios, sendo suspensa a estimulação forçada. $\mathrm{O}$ acompanhamento da frequência cardíaca, a qual manteve-se estável durante todo o procedimento, foi feito com a utilização de aparelho de ultrassom para observar a frequência da contratilidade do órgão.

A paciente foi posicionada em decúbito dorsal, sendo realizada antissepsia com iodopovidona (PVPI) em toda região do plastrão. Para a celiotomia foi feita uma incisão retangular no plastrão em ângulo de $30^{\circ} \mathrm{com}$ auxílio de uma pequena serra circular acoplada a um motor odontológico de baixa rotação, transpondo o tecido ósseo. Durante esse processo, o qual durou entre 30-40 minutos, realizou-se irrigação com solução fisiológica estéril a fim de dissipar o calor resultante e lavar os debris ósseos. Após a incisão completa do plastrão, realizou-se a divulsão da musculatura aderida ao periósteo (Figura 4). A janela óssea retirada do permaneceu protegida por compressa estéril e irrigada com solução fisiológica estéril durante todo o procedimento.

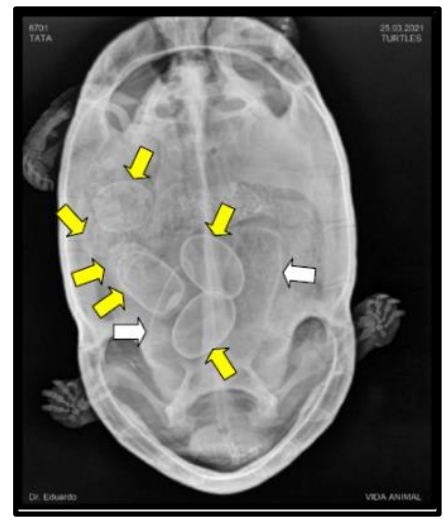

Figura 3. Exame radiográfico de controle durante a internação de jabuti-piranga na posição dorsoventral apresentando estruturas ovoides (seta amarela) e alças intestinais distendidas por gás (seta branca).

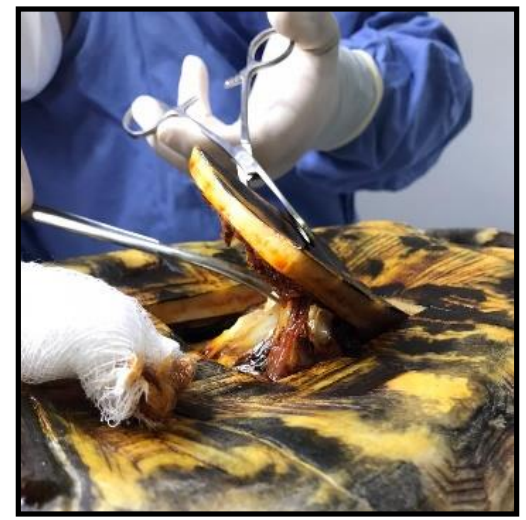

Figura 4. Incisão retangular em plastrão de jabutipiranga para celiotomia e ovariosalpingooviductotomia. 
Realizou-se a abertura do peritônio com auxílio de bisturi $\mathrm{n}^{\circ} 24$ e tesoura de metzembaum reta, evidenciando as estruturas internas e conteúdo líquido de aspecto turvo com presença de gema difusa na cavidade celomática, indicando o rompimento de óvulos em desenvolvimento (Figura 5).

Inicialmente foi feita a limpeza da cavidade celomática com auxílio de gaze e solução fisiológica estéreis. Posteriormente, as estruturas dos salpinges e ovários foram expostas manualmente, observando-se grande quantidade de folículos ovarianos em desenvolvimento (Figura 6), retirando-os e, posteriormente, realizando a hemostasia dos vasos que nutrem essas estruturas com ligadura utilizando fio de poliglactina 3-0.

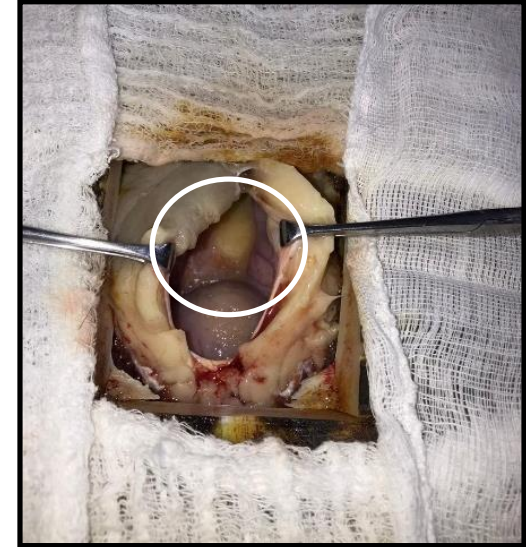

Figura 5. Conteúdo líquido de aspecto turvo com presença de gema difusa (círculo) na cavidade celomática de jabuti-piranga

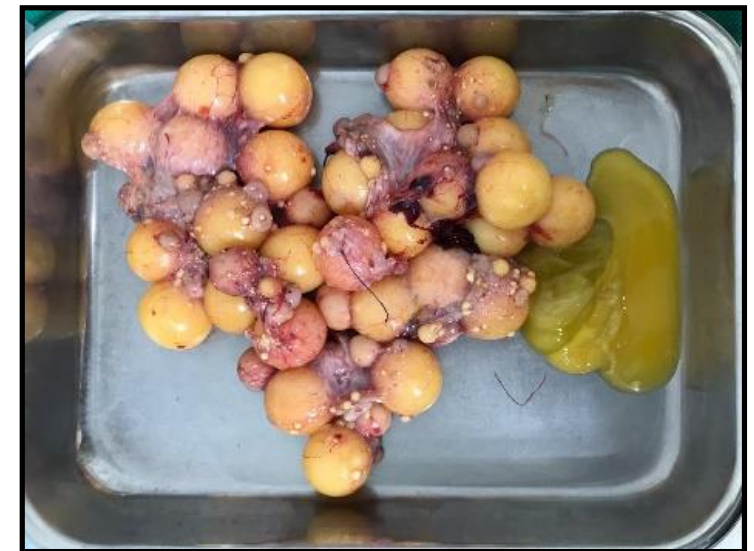

Figura 6. Folículos ovarianos em desenvolvimento apresentando diversos tamanhos e presença de um folículo rompido retirados de jabuti-piranga submetida à celiotomia e ovariosalpingooviductotomia.

Após a retirada completa dos óvulos em desenvolvimento presentes nas estruturas ovarianas e salpinges, expôs-se e realizou-se a secção do oviduto (Figura 7A), o qual continha 6 ovos no interior com tamanhos variados e defeitos na casca, como casca densa, um ovo de casca fina que foi rompida no processo de abertura do oviduto além de grande deposição de cálcio ao redor, caracterizando uma casca bastante áspera (Figura 7B). A excisão do órgão foi feita com auxílio de pinça hemostática semelhante ao procedimento cirúrgico de histerectomia. Posteriormente foi realizada sutura do tipo Parker-Kerr com fio de poliglactina 3-0 garantindo o fechamento da luz do órgão.

Após esse procedimento, lavou-se a cavidade celomática a fim de reduzir o conteúdo de óvulos rompidos. O fechamento do peritônio foi realizado com pontos isolados simples utilizando o fio de poliglactina 3-0. O completo isolamento da cavidade celomática foi testado com pressão positiva por meio de insuflamento do sistema respiratório da paciente.

A janela do plastrão foi reposicionada e, com auxílio de uma broca de aço inox, foram abertos quatro canais para passagem de fios de cerclagem 2-0. Os canais foram posicionados par a par no plastrão e no fragmento ósseo, sendo dois na porção cranial e dois na porção caudal da janela óssea. Finalmente esta foi fixada no plastrão por meio de fios de cerclagem 2-0 (igura 8).

Finalizando o procedimento, realizou-se curativo externo com PVPI e gaze estéril, fixado com esparadrapo comum.

Por fim, foi aplicado o reversor atipamezole $(0,7 \mathrm{mg} / \mathrm{kg})$ por via intramuscular e no pós-operatório imediato, fluidoterapia com ringer lactato $(40 \mathrm{~mL})$ por via subcutânea contendo uma solução de aminoácidos, vitaminas do complexo B, carboidratos, macro e microminerias (Mercepton ${ }^{\circledR}$ e Bionew ${ }^{\circledR}$ ) (ambos em dose empírica de $3 \mathrm{~mL} / \mathrm{animal}$ ). A paciente permaneceu entubada, sem oxigênio, até o dia seguinte e em ambiente aquecido e silencioso até total recuperação e retorno da anestesia.

Para o pós-operatório foi prescrito metronidazol $(12 \mathrm{mg} / \mathrm{kg})$ por via subcutânea, uma vez ao dia durante cinco dias, fluidoterapia com ringer lactato, pela via subcutânea, no volume de $40 \mathrm{~mL}$ contendo borogluconato de cálcio $(10 \mathrm{mg} / \mathrm{kg})$, enrofloxacina $(5 \mathrm{mg} / \mathrm{kg})$, butorfanol $(0,5 \mathrm{mg} / \mathrm{kg})$ e Mercepton ${ }^{\circledR}$ (dose empírica de $5 \mathrm{~mL} / \mathrm{animal}$ ), a cada dois dias, totalizando três aplicações e troca de curativo. 
A paciente recebeu alta médica sete dias após o procedimento, sendo recomendado apenas retorno para refazer o curativo da incisão cirúrgica a cada 3 dias por duas semanas, depois seguir com a troca do curativo em casa. Porém, a tutora decidiu deixar a paciente internada por mais um período. Assim, após 39 dias, a paciente retornou para o ambiente domiciliar, apresentando recuperação total.

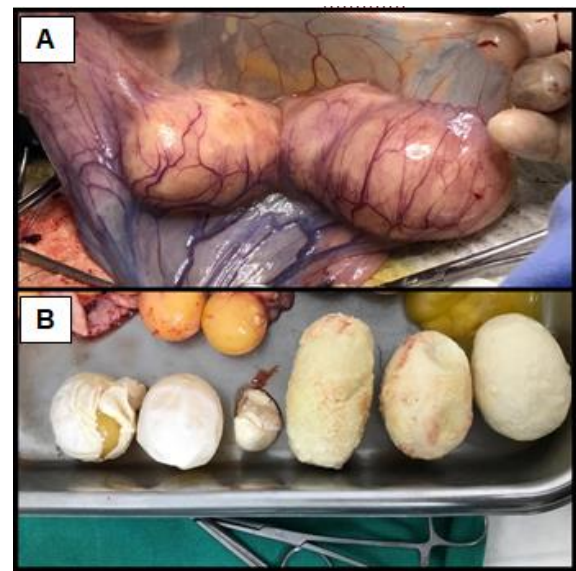

Figura 7. Celiotomia e ovariosalpingooviductotomia em jabuti-piranga. A) Exposição do oviduto contendo ovos no interior. B) Ovos densamente calcificados retirados do oviduto.

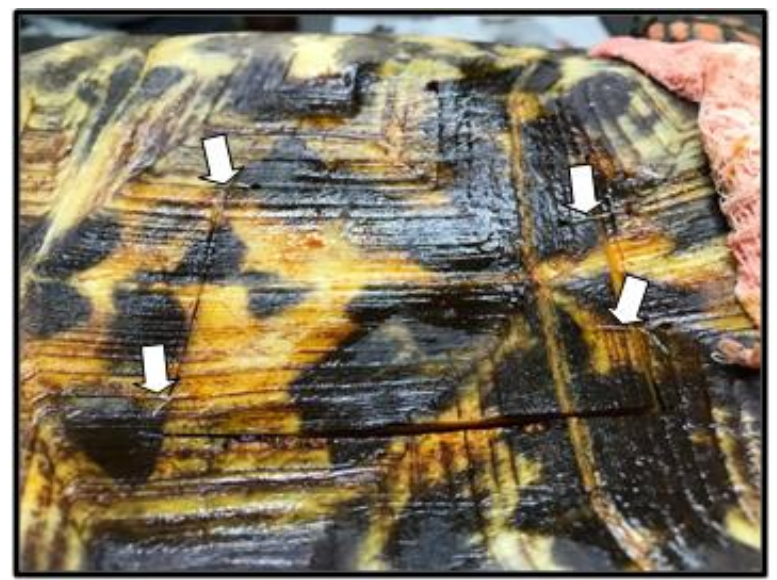

Figura 8. Janela óssea fixada novamente ao plastrão de jabutipiranga com fios de cerclagem 2-0 (seta) após procedimento cirúrgico de celiotomia e ovariosalpingooviductotomia.

\section{Discussão}

Segundo Matias et al. (2006), o diagnóstico definitivo de retenção de ovos em quelônios requer uma minuciosa investigação clínica, além da associação de exames complementares, como o radiográfico. De acordo com Denardo (1996), inicialmente pode haver dificuldade na diferenciação entre distocia e o desenvolvimento embrionário normal, por isso a importância de correlacionar a uma boa anamnese. No presente caso, o histórico de contrações contínuas da cloaca sem sucesso logo após a postura do primeiro ovo foi de grande importância para o diagnóstico presuntivo de distocia.

O exame de radiografia foi de extrema importância para a resolução do caso descrito, pois pode ser observado dois ovos irregulares e quatro ovos bem formados, além de casca hipercalcificada. Esse método de diagnóstico possibilita evidenciar o tamanho dos ovos, sua forma e posicionamento, assim como a possível hiper calcificação da casca (Matias et al., 2006).

Após o diagnóstico de distocia não obstrutiva, deve ser fornecido um ambiente correto, incluindo temperatura, umidade e câmaras de ninho apropriadas (terra ou areia) para poder induzir a deposição normal de ovos (Jepson, 2010), condições as quais foram fornecidas na clínica para a paciente descrita. Além disso, sugere-se a indução medicamentosa com cálcio e ocitocina para aumentar a força e frequência da contratilidade da musculatura do oviduto (Jepson, 2010). As doses de cálcio e ocitocina utilizadas neste relato foram baseadas no padrão apresentado na literatura para répteis. Há indicação de realizar 3 aplicações de ocitocina com intervalo de 90 minutos. Caso não haja postura, deve-se aumentar a dose, porém, no caso descrito, foi aplicada apenas uma dose a cada 3 dias, totalizando 3 aplicações. Isto foi realizado pensando no lento metabolismo dos répteis e também pelo risco de ruptura do oviduto. Também foi utilizada a menor dose de cálcio indicada devido a presença de calcificação dos ovos. Tendo em vista o sangramento que a paciente estava apresentando pela cloaca, foi administrado o antibacteriano enrofloxacina, pois há indicação para o tratamento de doenças infecciosas causadas por bactérias gram-positivas e gram-negativas (Viana, 2014).

O monitoramento radiográfico realizado na paciente durante o período de internação foi decisivo para a mudança de conduta, sendo necessária a intervenção cirúrgica. Este procedimento diagnóstico possibilita analisar a movimentação dos ovos durante o tratamento com ocitocina (Matias et al., 2006). O metronidazol prescrito após avaliação da segunda radiografia, na qual foi observada alteração nas alças intestinais, bem como no pós-operatório, devido a contaminação pela gema de ovo na cavidade 
celomática, foi com o intuito de combater bactérias anaeróbias e ser fortemente indicado em casos de infecções intestinais (Viana, 2014).

Observações de Mader (1996) indicam que as intervenções cirúrgicas são indicadas quando as técnicas clínicas não invasivas fracassarem na resolução de distocias ou quando existirem evidências de que a ovo postura não é possível. Denardo (1996) também afirma que, se os ovos apresentarem formatos anormais e cascas espessas, além da presença de secreções cloacais, os ovos devem ser removidos. Isto foi observado na paciente, que apresentou sangramento após a postura e presença de ovos densos e irregulares no exame de imagem, motivos pelos quais já se considerou a grande possibilidade de intervenção cirúrgica. Caso não fosse realizada a cirurgia, a paciente poderia apresentar um quadro de sepse decorrente do extravasamento de líquido dos folículos para a cavidade celomática, tendo a ovocelomite como resultado das gemas liberadas no interior da cavidade (Cubas et al., 2014), o que de fato aconteceu e foi evidenciando logo no início da celiotomia. Além disso, também haveria o risco de ruptura do oviduto, visto que os ovos já estavam com bastante deposição de cálcio em volta e gerando atrito à parede do órgão.

As medicações anestésicas utilizadas em répteis seguem as mesmas utilizadas em mamíferos, podendo haver alterações em doses e efeitos (Mosley, 2006). Para a MPA da paciente, utilizou-se agentes anestésicos associados, entre eles a cetamina, que se trata de um anestésico dissociativo comumente utilizado como parte do protocolo anestésico em répteis, com o objetivo de produzir imobilização e induzir a anestesia (Mader, 1996). Foram associados outros agentes como o midazolam, um benzodiazepínico pré-anestésico indicado para promover, entre outras ações, o miorrelaxamento (Fantoni \& Cortopassi, 2009; Fantoni \& Mastrocinque, 2005). Associou-se também butorfanol, um analgésico opioide sedativo e também pré-anestésico, e dexmedetomidina, que se trata de um agonista alfa2-adrenérgico analgésico, sedativo e adjuvante anestésico. O propofol, alquifenol sedativo e anestésico geral de curta duração, foi utilizado para indução da anestesia. Se tratando de répteis, é indicado antagonizar a dexmedetomidina a fim de diminuir o tempo de recuperação anestésica. Sendo assim, foi aplicado o fármaco atipamezole, um antagonista alfa 2-adrenérgico reversor dos efeitos de diversos fármacos, incluindo a dexmedetomidina, a qual foi administrada na paciente do presente relato (Viana, 2014).

Segundo Dutra (2014), para a cirurgia celomática, em muitos quelônios a osteotomia do plastrão é necessária. $\mathrm{O}$ autor ainda afirma que é necessário algum tipo de serra específica para ossos para atravessar o plastrão ósseo. Procedimentos de tecido mole dentro da cavidade celomática são realizados com protocolos similares aos utilizados em mamíferos (Dutra, 2014). Suturas com categute cromado são evitadas, pois promovem grande resposta inflamatória (Dutra, 2014). Ressalta-se o uso de fio de poliglactina na paciente relatada.

Quando a celiotomia acaba, a cavidade deve ser lavada com solução fisiológica, removendo qualquer detrito de casco ou outras contaminações. A linha medioventral deve ser fechada com padrão simples contínuo ou simples com fio de material absorvível (Mader, 1996). Na paciente descrita, a lavagem da cavidade também foi necessária para remover resíduos foliculares remanescentes. $\mathrm{O}$ fechamento da cavidade foi realizado com pontos simples isolados e fio de poliglactina 3-0.

Em cirurgias obstétricas, como a ovariosalpingooviductotomia, em alguns casos está presente grande quantidade de folículos ovarianos. Nos casos de folículos pré-ovulatórios, os ovários deverão ser removidos em primeiro lugar, enquanto que, em casos de folículos pós-ovulatórios, em que há ovos dentro do oviduto, os ovos devem ser primeiramente retirados, permitindo assim maior acessibilidade aos ovários (Dutra, 2014). Neste relato não foi possível realizar nesta ordem exata, visto a grande quantidade de folículos presentes impedia a visualização do oviduto, além da possibilidade de rompimento folicular.

A presença de gema de ovo no interior da cavidade celomática induz a uma grave reação inflamatória. O tratamento consiste na remoção do material e irrigação abundante com solução fisiológica estéril da cavidade celomática, além de antibioticoterapia sistêmica, manutenção da hidratação e equilíbrio nutricional (Denardo, 1996; Pessoa et al., 2008). Concordando com a literatura, na paciente descrita realizou-se a lavagem da cavidade celomática com a finalidade de reduzir o conteúdo de folículos rompidos e foi prescrita antibioticoterapia e fluidoterapia para o pós-cirúrgico. 
Os dois pontos mais importantes no pós-operatório são calor e controle da dor. Durante a recuperação anestésica, o paciente deve ser mantido em um ambiente limpo, escuro e silencioso. A fluidoterapia é indicada para manter a hidratação e volemia (Dutra, 2014), sendo todos esses fatores fornecidos para a paciente do caso relatado. Além disso, é de grande importância monitorar o processo de cicatrização da incisão cirúrgica, uma vez que a recuperação total do plastrão requer um longo período de regeneração até a completa ossificação, podendo ser de meses a anos (Cubas et al., 2014). Na paciente descrita, a lesão encontrava-se estável no momento da alta, já havendo iniciado o processo de calcificação da ferida cirúrgica.

As distocias nos quelônios de cativeiro podem estar associadas a erros de manejo ambiental e alimentar (Jones et al., 2016; Mitchel \& Tully, 2009; Schumacher, 2007). Sabe-se também que o período de postura dos quelônios varia entre os meses de setembro a novembro (Vogt et al., 2009). Neste caso, relacionou-se como causa principal da distocia, problemas climáticos. Tendo em vista que, o clima da cidade não era favorável para reprodução de jabutis, por ser a primeira vez que o animal realizava postura em 22 anos e os sinais clínicos surgiram no mês de março. Além disso, o ambiente e a alimentação da paciente descritas pela tutora não apresentavam falhas. A hiper calcificação dos ovos também foi fator importante para o impedimento da saída espontânea, sugerindo que a estase dos ovos ocorria há bastante tempo.

Zwart \& Vorstenbosch (1995) descrevem que anormalidades na casca dos ovos de répteis são frequentes e que a sua ocorrência implica sérias consequências para a viabilidade e a taxa de nascimento. Ovos de tamanho variado e com defeitos de casca, como áspera ou fina, deposição de cálcio em volta dos poros e ovos com múltiplas camadas são algumas das variações morfológicas descritas como anômalas em quelônios, as quais podem dificultar a ovo posição, características observadas no presente relato.

\section{Conclusão}

A realização de um exame clínico de qualidade associado ao exame radiográfico foi fundamental para a resolução do caso de distocia relatado. Inicialmente instituiu-se tratamento medicamentoso indicado pela literatura, porém, como a condição clínica da paciente não evoluiu, foi necessário intervir cirurgicamente, conforme recomendação de diversos autores, obtendo resultado satisfatório. A distocia em quelônios é uma condição patológica comumente atendida na clínica de animais silvestres, portanto, é dever do Médico Veterinário orientar o tutor a respeito de técnicas de manejo e alimentação corretas.

\section{Referências bibliográficas}

Cubas, Z. S., Silva, J. C. R., \& Dias, J. L. C. (2014). Tratado de animais selvagens-medicina veterinária. Editora Roca.

Denardo, D. (1996). Reproductive biology. In D R Mader (Ed.), Reptile medicine and surgery (p. 512 p.). W.B. Saunders Co.

Divers, S. J., \& Mader, D. R. (2005). Reptile medicine and surgery. Elsevier Health Sciences.

Dutra, G. H. P. (2014). Testudines (Tigre d'água, Cágado e Jabuti). In Z. S. Cubas, J. C. R. Silva, \& J. L. Catão-Dias (Eds.), Tratado de Animais Selvagens (pp. 219-258). Roca, Brasil.

Fantoni, D. T., \& Cortopassi, S. R. G. (2009). Anestesia em cães e gatos. Roca.

Fantoni, D. T., \& Mastrocinque, S. (2005). Analgesia preventiva. In P. E. Otero (Ed.), Dor: Avaliação e tratamento Mader,em pequenos animais (pp. 76-80). Interbook.

Jepson, L. (2010). Jabutis e cágados. In L. Jepson (Ed.), Clínica de Animais Exóticos: referência rápida. Sauders Elsevier.

Jones, K., Ariel, E., Burgess, G., \& Read, M. (2016). A review of fibropapillomatosis in green turtles (Chelonia mydas). The Veterinary Journal, 212, 48-57. https://doi.org/10.1016/j.tvj1.2015.10.041.

Mader, Douglas R. (1996). Reptile Medicine and Surgery-E-Book. Elsevier Health Sciences.

Matias, C. A. R., Romão, M. A. P., Tortelly, R., \& Bruno, S. F. (2006). Fisiopathological aspects of egg retention in South American Red-footed Tortoise (Geochelone carbonaria Spix, 1824). Ciência Rural, 36(5), 1494-1500. https://doi.org/10.1590/S0103-84782006000500023. 
Mitchel, M. L., \& Tully, T. N. (2009). Manual of exotic pet practice. Elsevier Science Healt h Science Division. https://doi.org/10.1016/B978-1-4160-0119-5.X5001-X.

Mosley, C. A. (2006). Anatomic and physiologic considerations for reptile anesthesia. Proceedings of the North American Veterinary Conference, 1643-1645.

Pessoa, C. A., Rodrigues, M. A., Kozu, F. O., Prazeres, R. F., \& Fecchio, R. S. (2008). Ooforectomia videoassistida por acesso pré-femural em targaruga-de-ouvido-vermelho (Trachemys scripta elegans). Pesquisa Veterinária Brasileira, 28, 345-349. https://doi.org/10.1590/S0100736X2008000700005.

Pritchard, P., Ernest, C. H., \& Barbour, R. (1990). Turtles of the world. Copeia, 2(602). https://doi.org/10.2307/1446379

Schumacher, J. (2007). Chelonians (Turtles, tortoises and terrapins). In G. West, D. Heard, \& N. Caukett (Eds.), Zoo animal and wildlife immobilizations and anesthesia. Blackwell Publishers Inc.

Viana, F. A. B. (2014). Guia terapêutico veterinário. In Lagoa Santa.

Vogt, R. C., Ferrara, C. R., Schneider, L., \& Junior, L. B. S. (2009). Brazilian Amazon turtles: habitat. Herpetological Review, 40(2), 213.

Zwart, P., \& Vorstenbosch, C. J. A. H. V. (1995). Abnormalities of the shell of eggs in reptiles. Herpetopathologia. Fifth International Colloquiem of Pathology of Reptiles, 233-238.

Histórico do artigo:

Recebido: 10 de outubro de 2021

Aprovado: 11 de novembro de 2021

Disponível online: 31 de dezembro de 2021
Licenciamento: Este artigo é publicado na modalidade Acesso Aberto sob a licença Creative Commons Atribuição 4.0 (CC-BY 4.0), a qual permite uso irrestrito, distribuição, reprodução em qualquer meio, desde que $o$ autor e a fonte sejam devidamente creditados. 